\title{
Flaring activity of microquasars from multi-frequency daily monitoring program with RATAN-600 radio telescope
}

\author{
S. A. Trushkin, N. A. Nizhelskij, N. N. Bursov and E. K. Majorova \\ Special Astrophysical Observatory RAS, Nizhnij Arkhyz, 369167, Russia \\ email: satr@sao.ru
}

\begin{abstract}
We report about the multi-frequency (1-30 GHz) daily monitoring of the radio flux variability of the three microquasars: SS433, GRS1915+105 and Cyg X-3 during 2005-2006. After a quiescent radio emission we have detected a drop down of the fluxes $(\sim 20 \mathrm{mJy})$ from Cyg X-3, a sign of the following bright flare, and indeed a 1 Jy flare was detected on 2 February 2006 after 18 days of quenched radio emission. The daily spectra of the flare in the maximum was found flat from 2 to $110 \mathrm{GHz}$, using the quasi-simultaneous observations at $109 \mathrm{GHz}$ with the RT45m telescope and the NMA millimeter array of Nobeyama Radio Observatory in Japan. Several bright radio flaring events $(1-15 \mathrm{Jy})$ followed during the state of highly variable and intense 1-12 keV X-ray emission ( 0.5 Crab), monitored in the RXTE ASM program. We discussed various spectral and temporal characteristics of the detected 180 day light curves from three microquasars in comparison with the Rossi XTE ASM data.
\end{abstract}

Keywords. X-rays: binaries, radio continuum: stars, radiation mechanisms: nonthermal

The two-side relativistic jets, collimated high-velocity outflows, ejected from polar regions of accretion disks around black holes or neutron stars in the microquasars are efficient sources of variable synchrotron emission. Light curves and spectra variations are a key probe to test models of cosmic jets. We have carried out the 250-day monitoring of the microquasars Cyg X-3, GRS 1915+105, and SS433, with RATAN-600 radio telescope at 1-30 GHz from September 2005 to May 2006. Trushkin et al. (2007a) discussed the detected X-ray/radio light curves correlation from GRS 1915+105.

We have used a continuum radiometer complex: the four cryo-receivers at $3.9,7.7$, 11.2 , and $21.7 \mathrm{GHz}$ and three low-noise transistor radiometers at 1, 2.3 and $30 \mathrm{GHz}$. The observations were carried out with the 'Northern sector' antenna of RATAN-600.

During 100 days, Cyg X-3 was in a quiescent state of 100 mJy (Fig. 1). In December 2005 its X-ray and radio fluxes began to increase. Then the flux density at $4.8 \mathrm{GHz}$ was found to drop from 103 mJy on Jan 14.4 (UT) to 22 mJy on Jan 17.4 (UT). The source is known to exhibit the radio flares typically with a few peaks exceeding $1-5$ Jy following such a quenched state as Waltman et al. (1994) have showed in the intensive monitoring of Cyg X-3 at 2.25 and 8.7 GHz. The source has been monitored from Jan 25 (UT) with the Nobeyama Radio Observatory 45m Telescope and the Nobeyama Millimeter Array (NMA). On Feb 2.2 (UT), about 18 days after it entered the quenched state, the rise of a first peak is detected with the NRO45m Telescope. On Feb 3.2 (UT), the flux densities reached to the first peak at all the sampling frequencies from $2.25 \mathrm{GHz}$ to $110.10 \mathrm{GHz}$ (Tsuboi et al. 2006). The spectrum on Feb 03 of the flare was flat as measured by RATAN, NRO RT45m and NMA from 2 to $110 \mathrm{GHz}$. The next peak of the active events on 10 February have a flat spectrum at a level of 1Jy again. Later three short-duration flares have happened during a week. A flare on Feb 18 had the inverted power-law spectrum with the spectral index $\alpha=+0.75$ from 2.3 to $22 \mathrm{GHz}$. 


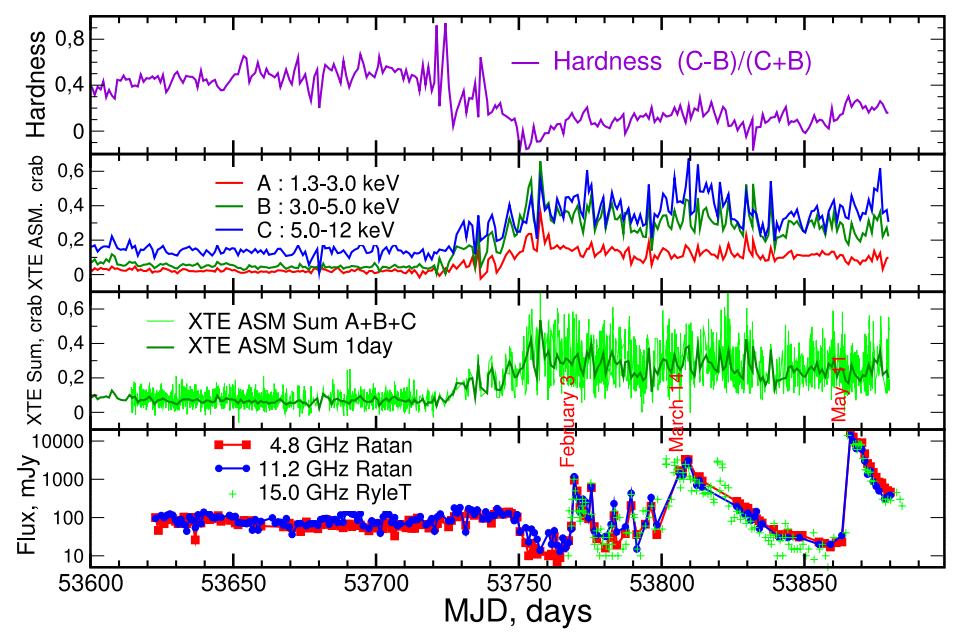

Figure 1. The RATAN and RXTE ASM light curves of Cyg X-3, September 2005 - May 2006.

We detected three powerful flares: on March 14 to $5 \mathrm{Jy}$, on May 11 to $16 \mathrm{Jy}$ and on July 26 to $13 \mathrm{Jy}$. In the May flare fluxes have grown up by a factor $\sim 1000$ during a one day. The change of the spectrum during the flare on May 11-19 followed to model of single ejection of the relativistic electrons, moving in thermal matter in a intense WR star wind. It was in a optically thin mode at frequencies $2-22 \mathrm{GHz}$, meanwhile at 614 $\mathrm{MHz}$ (Pal et al. 2006) and $1 \mathrm{GHz}$ Cyg X-3 was in a hard absorption.

The first microquasar SS433, a bright variable emission star, supercritical accreting X-ray binary, identified with a radio source $1909+048$ has an unresolved compact radio core and 1 arcsec long aligned jets, as discovered with the MERLIN system. Kotani et al. (2006) detected the fast variation in the X-ray emission of SS433 during the radio flares, probably even QPOs of $0.11 \mathrm{~Hz}$. In Trushkin et al. (2007b) the daily RATAN light curves are given. In the bright flare of Feb 16, 2006 we detected a delay of the maximum flux at $1 \mathrm{GHz}$ about 2 days, and 1 day at $2.3 \mathrm{GHz}$, respectively, relatively the maxima at $n u>4 \mathrm{GHz}$. Some flares happened just close to the multi-band program of the studies of SS433 in 3-6 April 2006 (Kotani et al. 2007).

\section{Acknowledgements}

These studies were supported by the Russian Foundation Basic Research (RFBR) grant N 05-02-17556 and by the mutual RFBR and Japan Society for the Promotion of Science (JSPS) grant N 05-02-19710.

\section{References}

Kotani, T., Trushkin, S. A., Valiullin, R. K. et al. 2006, ApJ, 637, 486

Kotani, T., Trushkin, S. et al. 2007, VI microquasars workshop, Komo, Italy, PoS, (in press) Levine, A., Bradt, H., Cui, W. et al. 1996, ApJ, 469, L33

Pal, S., Ishwara-Chandra, C. H. \& Pramesh, A. 2006, ATel, \#809

Tsuboi, M., Kuno, N., Umemoto, T. et al. 2006, ATel, \#727, 1

Trushkin, S., Kotani, T. et al. 2007a, in this Volume

Trushkin, S., Bursov, N. et al. 2007b, VI microquasars workshop, Komo, Italy, PoS, in press

Waltman, E. B., Fiedler, R. L., Johnston, K. L. \& Ghigo, F. D. 1994, AJ, 108, 179 